\title{
Oocyte Cryopreservation before Tumorectomy in Gorlin-Goltz Syndrome with Recurrent Ovarian Fibroma: A Case Report
}

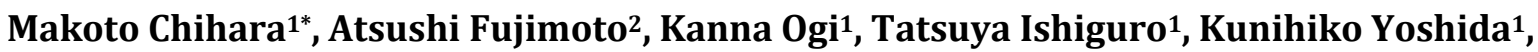 \\ Masayuki Sekine', Koich Takakuwa1, Hiroki Fujikawa², Yutaka Shimomura ${ }^{3}$, \\ Takayuki Enomoto ${ }^{1}$
}

\footnotetext{
${ }^{1}$ Department of Obstetrics and Gynecology, Niigata University Graduate School of Medical and Dental Sciences, Niigata, Japan ${ }^{2}$ Department of Dermatology, Niigata University Graduate School of Medical and Dental Sciences, Niigata, Japan ${ }^{3}$ Department of Dermatology, Yamaguchi University Graduate School of Medicine, Yamaguchi, Japan

Email: *chihara-m@med.niigata-u.ac.jp
}

How to cite this paper: Chihara, M., Fujimoto, A., Ogi, K., Ishiguro, T., Yoshida, K., Sekine, M., Takakuwa, K., Fujikawa, H., Shimomura, Y. and Enomoto, T. (2018) Oocyte Cryopreservation before Tumorectomy in Gorlin-Goltz Syndrome with Recurrent Ovarian Fibroma: A Case Report. Open Journal of Obstetrics and $G y$ necology, 8, 836-846.

https://doi.org/10.4236/ojog.2018.810087

Received: July 10, 2018

Accepted: August 20, 2018

Published: August 23, 2018

Copyright (c) 2018 by authors and Scientific Research Publishing Inc. This work is licensed under the Creative Commons Attribution International License (CC BY 4.0).

http://creativecommons.org/licenses/by/4.0/

\begin{abstract}
Background: Gorlin-Goltz syndrome is a very rare syndrome that reportedly occurs in 1 of 235,800 people in Japan. The proportion of women with Gorlin-Goltz syndrome complicated with an ovarian fibroma ranges from $12.5 \%$ to $17.0 \%$. Some surgical cases of Gorlin-Goltz syndrome with an ovarian tumor have been reported. However, no studies have mentioned subsequent fertility preservation by using oocyte cryopreservation process. Case: In this case report, the patient with Gorlin-Goltz syndrome underwent laparotomy for bilateral ovarian fibrothecoma at 15 years of age. At 20 years of age, a recurrent ovarian tumor was detected in the remaining ovary. During the follow-up, we detected an increase in its size. As tumor torsion requiring left salpingo-oophorectomy was possible, tumorectomy was considered. However, her anti-Müllerian hormone level was low. As she was at risk for premature ovarian failure after tumorectomy, we planned to cryopreserve her oocytes to preserve her fertility before tumorectomy. Outcome: When the patient underwent surgery, the diameter of her left ovarian tumor was found to have increased to $56 \mathrm{~mm}$. Egg collection was performed twice, and two oocytes were cryopreserved. Subsequently, she underwent tumorectomy of the left ovarian tumor. No recurrence has been observed. Conclusion: If a recurrent ovarian tumor is detected in patients with Gorlin-Goltz syndrome and a low anti-Müllerian hormone level, cryopreservation of oocytes before tumorectomy may be effective for preserving their fertility.
\end{abstract}




\section{Keywords}

Case Reports, Cryopreservation, Fibroma, Gorlin-Goltz Syndrome, Recurrence

\section{Introduction}

Gorlin-Goltz syndrome, which is a neurodermal syndrome, was first reported by Gorlin and Goltz in 1960 [1]. It is also called nevoid basal cell carcinoma syndrome [1] [2]. This syndrome is an autosomal dominant disorder [2] [3], and it is associated with chromosome 9q22.3-q31 [4].

In 1996, it was reported that the causative gene of nevoid basal cell carcinoma syndrome is the human homolog of the Drosophila patched gene $P T C H 1$, which is a receptor of the hedgehog protein [5]. To date, some PTCH1 mutations have been identified in patients with Gorlin-Goltz syndrome [6]. In Japan, the Gorlin-Goltz syndrome is reported to occur in 1 in 235,800 people [7].

Gorlin-Goltz syndrome is a disease characterized by developmental anomalies, such as palmar pits and rib anomalies, and tumorigenesis, such as medulloblastoma and basal cell carcinoma [8]. The diagnostic criteria for Gorlin-Goltz syndrome were proposed by Kimonis et al. of the National Institutes of Health in the United States, and were composed of six major and six minor criteria (Table 1) [9]. Among these criteria, the proportion of women aged 16 45 years with an ovarian fibroma ranged from $12.5 \%$ to $17.0 \%$ [7] [9]. Some surgical cases of Gorlin-Goltz syndrome with an ovarian tumor have been reported [10] [11]. One study reported the use of conservative treatment for recurrent ovarian fibromas in a 22-year-old patient with Gorlin-Goltz syndrome [10], and the other described a case of Gorlin-Goltz syndrome complicated with hydronephrosis due to a huge ovarian tumor [11]. However, no studies have mentioned subsequent fertility preservation by using oocyte cryopreservation process.

We report on a case of Gorlin-Goltz syndrome in a patient who underwent oocyte cryopreservation before tumorectomy. Herein, we describe the patient's treatment course and provide a literature review.

\section{Case Report}

A 20-year-old woman (unmarried, gravida 0) with Gorlin-Goltz syndrome was referred to the Department of Obstetrics and Gynecology of Niigata University Graduate School Medical and Dental Science, Niigata, Japan for follow-up of a recurrent ovarian tumor and future preservation of fertility.

\subsection{Medical History}

The patient had delayed physical development during infancy; it took her 1 year, 8 months to walk, but eventually, she caught up with her age group. From early 
Table 1. Diagnostic criteria for nevoid basal cell carcinoma syndrome ${ }^{\mathrm{a}}$.

A diagnosis of NBCC is made in the presence of two major or one major and two minor criteria

Major criteria

1) More than two BCCs or one BCC in a patient younger than 20 years

2) Odontogenic keratocysts of the jaw bone proven by histological findings

3) Three or more palmar or plantar pits

4) Bilamellar calcification of the falx cerebri

5) Bifid, fused, or markedly splayed ribs

6) First-degree relative with NBCC syndrome

Minor criteria

Any one of the following features:

1) Macrocephaly determined after adjustment for height

2) Congenital malformations: cleft lip or palate, frontal bossing, coarse facial features ("coarse face"), and moderate or severe hypertelorism

3) Other skeletal abnormalities: Sprengel deformity, marked pectus deformity, marked syndactyly of the digits

4) Radiological abnormalities: bridging of the sella turcica, vertebral anomalies such as hemivertebrae, fusion or elongation of the vertebral bodies, modeling defects of the hands and feet, or flame-shaped lucencies of the hands or feet

5) Ovarian fibroma

6) Medulloblastoma

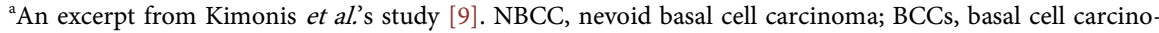
mas.

childhood, palmar pits were conspicuous during bathing, but she had no other symptoms.

At 15 years of age, she developed acute abdomen and was diagnosed as having right ovarian tumor stem torsion, and a left ovarian tumor was detected by transvaginal ultrasonography and computed tomography (CT). Emergent right salpingo-oophorectomy and left ovarian tumorectomy were performed. Fibrothecoma was diagnosed on the basis of the pathological tissue findings.

At 16 years of age, she underwent corrective surgery for strabismus of both eyes. At 19 years of age, she visited the dentist who incidentally detected discharge from a jawbone cyst. Radiography confirmed the presence of multiple cysts in the upper and lower jawbones. Results of histopathological examination of the cyst indicated odontogenic keratosis. A high possibility of Gorlin-Goltz syndrome was considered because of her surgical history for an ovarian tumor and the findings of coarse facial features, macrocephaly, palmar pits, and odontogenic keratosis. Thus, she was referred to the Department of Dermatology in our hospital for evaluation of the palmar pits and possible diagnosis of basal cell carcinoma.

A head CT examination was performed, and calcification of the falx cerebri was detected (Figure 1). Further, pseudopits and keratotic lesions with central depressions were observed on both palms (Figure 2(a)), and skin biopsy results of a specimen obtained from a pigmented lesion of the left sole showed 
well-circumscribed collections of basophilic basaloid cells in the dermis, with peripheral nuclear palisading and surrounding mucinous stroma (Figure 2(b)).

$P T C H 1$ gene analysis was performed using genomic deoxyribonucleic acid extracted from peripheral blood, and a heterozygous mutation c.2369_2370delTT (p.F790Yfs ${ }^{*} 38$ ) was identified in exon 15 of the $P T C H 1$ gene. On the basis of the aforementioned results and the patient's medical history, a diagnosis of Gorlin-Goltz syndrome was confirmed. Her brother's and parents' genes were not analyzed, as they did not show any symptoms.

\subsection{Clinical Course}

The dermatologist at our hospital advised the patient that recurrence of the ovarian tumor was possible and recommended a visit to the gynecologist who performed the initial surgery. As anticipated, recurrence of the ovarian tumor was detected 5 years postoperatively by transvaginal ultrasonography.

The patient also underwent magnetic resonance imaging. A left ovarian tumor with a diameter of $36 \mathrm{~mm}$ was detected. The tumor had a low signal intensity on T1-weighted/T2-weighted imaging, and a solid lesion was detected with contrast-enhanced imaging. Benign tumors, such as a fibroma and thecoma, were suspected (Figure 3). Because the recurrent ovarian tumor was also thought to be affected by Gorlin-Goltz syndrome, a multidisciplinary treatment approach was considered appropriate. The patient was referred to the Department of Obstetrics and Gynecology of Niigata University Graduate School Medical and Dental Science, Niigata, Japan, for follow-up of the ovarian tumor. In addition, she hoped for a second opinion about preservation of fertility in the future.

\subsection{Laboratory Findings}

Findings of the pelvic examination showed that the left ovarian tumor was palpable and mobile. The solid, left ovarian tumor measured $25 \mathrm{~mm}$ with a 20-mm diameter, as detected by transvaginal ultrasonography.

Basal hormone data were as follows: prolactin level, $12.3 \mathrm{ng} / \mathrm{mL}$; luteinizing hormone level, $3.7 \mathrm{mIU} / \mathrm{mL}$; follicle-stimulating hormone level, $9.2 \mathrm{mIU} / \mathrm{mL}$; estradiol level, $<20 \mathrm{pg} / \mathrm{mL}$; testosterone level, $18.47 \mathrm{ng} / \mathrm{dL}$; and anti-Müllerian hormone (AMH) level, $1.53 \mathrm{ng} / \mathrm{mL}$.

The findings did not indicate that surgical intervention was required; hence, follow-up was planned. However, the tumor size increased to $56 \mathrm{~mm}$, and the tumor volume increased during the follow-up (Figure 4).

If the tumor became twisted again, salpingo-oophorectomy would be necessary, and the patient would be at possible risk of loss of fertility. Hence, we obtained the patient's informed consent to perform tumorectomy. The risk of premature ovarian failure after tumorectomy was also considered. Therefore, we performed oocyte cryopreservation to her preserve fertility before tumorectomy.

This disease has autosomal dominant inheritance [2] [3], so we explained to the patient the possibility that $50 \%$ of her children derived from cryopreserved 


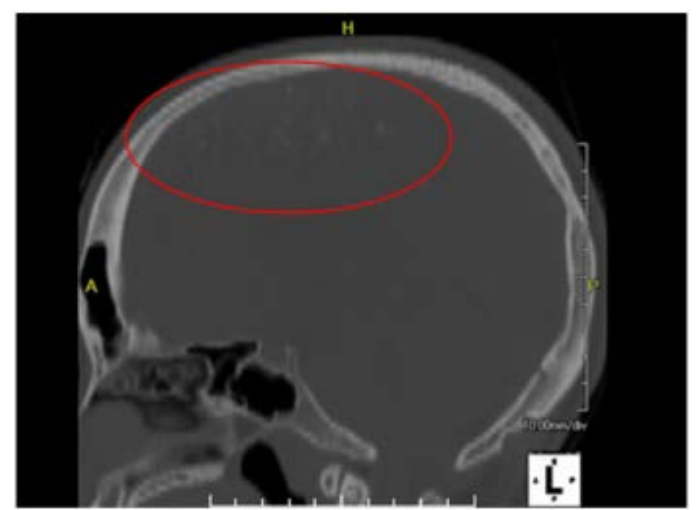

Figure 1. Head computed tomography scan showing calcification of the falx cerebri.
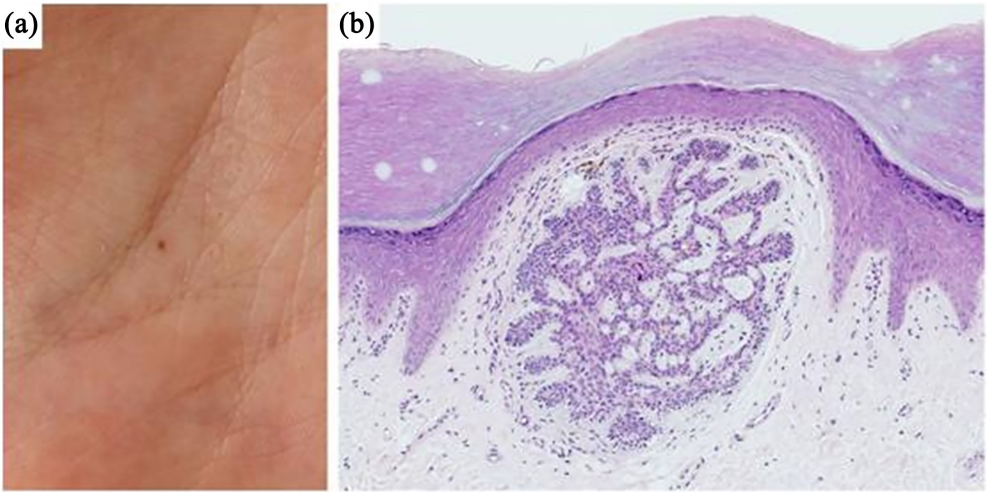

Figure 2. Clinical and histological features of Gorlin-Goltz syndrome in our patient. (a) Pseudopits and keratotic lesions with central depressions are present on both palms; (b) Results of the skin biopsy specimen from a pigmented lesion of the left sole show well-circumscribed collections of basophilic basaloid cells in the dermis, with peripheral nuclear palisading and surrounding mucinous stroma (hematoxylin and eosin staining; original magnification, $\times 100)$.

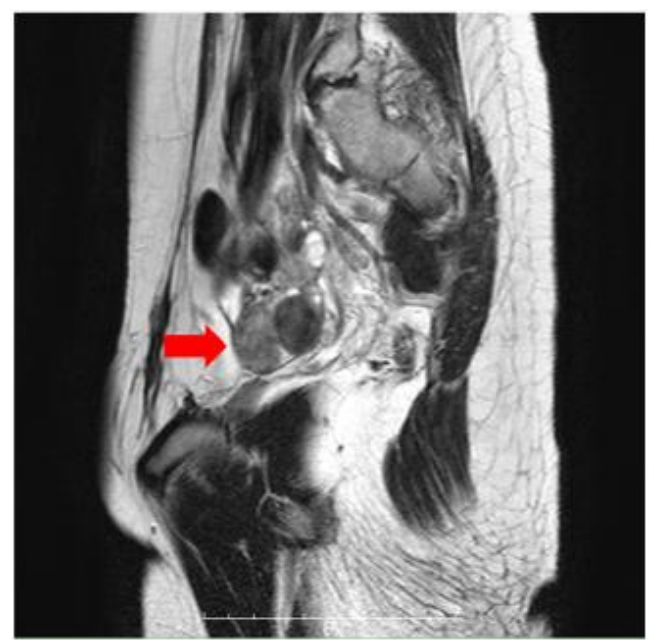

Figure 3. Left ovarian tumor visible on a T2-weighted magnetic resonance imaging scan. The tumorous lesion measures $36 \mathrm{~mm}$ in diameter, has a low T2-weighted intensity, and is located in the left ovary (arrow). 


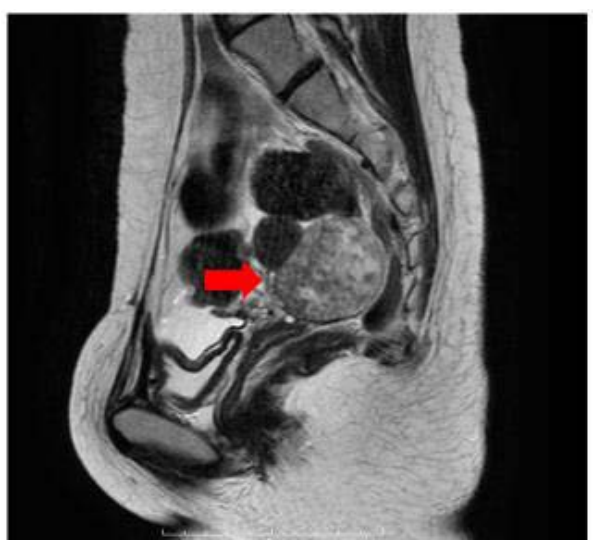

Figure 4. Left ovarian tumor visible on a T2-weighted magnetic resonance imaging scan. The tumorous lesion measures $56 \mathrm{~mm}$ in diameter, has a T2-weighted low intensity, and is located in the left ovary (arrow).

oocytes in assisted reproductive technology could develop Gorlin-Goltz syndrome. She also provided consent to undergo oocyte cryopreservation. Additionally, oocyte cryopreservation for this patient was approved by the Clinical Ethical Committee of Niigata University Medical and Dental Hospital.

The short protocol was chosen as the controlled ovarian stimulation protocol for egg collection. On the first attempt, from day 5 of the menstrual cycle, $150 \mathrm{U}$ of human menopausal gonadotropin (hMG) was intramuscularly injected daily. The administration of nasal drops of gonadotropin-releasing hormone ( $\mathrm{GnRH})$ agonist ( $900 \mu \mathrm{g} /$ day; Suprecur, Mochida Pharma) was started at the same time. After $1200 \mathrm{U}$ of hMG was injected intramuscularly, we confirmed the presence of four mature follicles measuring $15 \mathrm{~mm}$ or more. Additionally, 10,000 $\mathrm{U}$ of human chorionic gonadotropin was injected intramuscularly as a trigger. After 34 hours, we transvaginally punctured the four mature follicles with an 18-gauge (G) needle and collected one metaphase stage II (MII) egg. On the same day, we cryopreserved an MII oocyte by using the ultra-rapid vitrification method (Cryotop Safety Kit Protocol, Cryotop Method for VT505-TOP-KIT, Kitazato Corporation).

Subsequently, the patient hoped to undergo oocyte cryopreservation one more time as only one frozen egg was preserved the first time. Using the same method, we induced ovulation by using the short protocol. After $1800 \mathrm{U}$ of hMG was injected intramuscularly, we confirmed the presence of two mature follicles measuring $18 \mathrm{~mm}$ or more. On the next day, 10,000 $\mathrm{U}$ of human chorionic gonadotropin was injected intramuscularly as a trigger. After 34 hours, two mature follicles were punctured transvaginally by an $18-\mathrm{G}$ needle, and we collected an MII egg. On the same day, we cryopreserved the MII oocyte in the same manner as mentioned previously.

At 22 years of age, the patient underwent tumorectomy of the left ovarian tumor by laparotomy. The final histopathological diagnosis of the tumor was a fibroma (Figure 5). Postoperatively, menstruation returned to normal, and we observed antral follicles by transvaginal ultrasonography. 


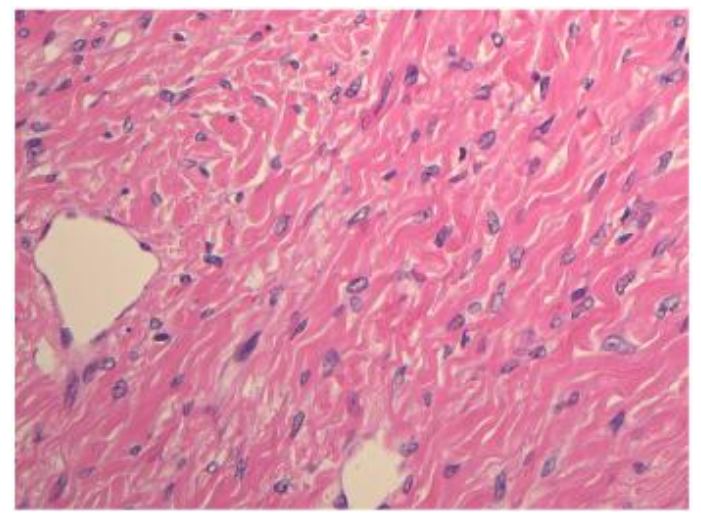

Figure 5. Final histopathological findings of the ovarian tumor. The tumor specimen shows fibroblasts and fibrocytes (hematoxylin and eosin staining; original magnification, $\times 400)$. The tumor was diagnosed as a fibroma.

Currently, the patient visits the gynecologist as an outpatient once every 6 months. No recurrence of the ovarian tumor has been observed, and the patient is still not pregnant.

\section{Discussion}

Regarding diagnostic criteria for Gorlin-Goltz syndrome, Kimonis et al.'s study is often quoted. They reported that the diagnosis of Gorlin-Goltz syndrome can be made when two of the six major criteria or one major and two of the six minor criteria are met [9]. In our case, the patient satisfied the three major criteria (odontogenic keratocysts of the jaw, three or more palmar pits, and bilamellar calcification of the falx cerebri) and three minor criteria (macrocephaly, coarse facial features, and ovarian fibroma). Her brother and parents did not show any of the aforementioned symptoms; hence, the mutations identified in this case are most likely de novo mutations. However, it is unknown at which stage the mutation occurred. Because Gorlin-Goltz syndrome has various symptoms, as shown in our case, it tends to be diagnosed after the patient has visited several departments.

Generally, an ovarian fibroma can develop at any age, but it most frequently develops during middle age, at an average age of 48 years; fewer than $10 \%$ of the cases are encountered in patients younger than 30 years [12]. The onset of ovarian fibrothecoma in our case was 15 years of age, which was early. This may be an effect of Gorlin-Goltz syndrome.

Recurrence was detected at 5 years postoperatively in our patient (at 20 years of age). There are other reports of recurrent cases of ovarian tumors in young patients with Gorlin-Goltz syndrome [10], and preservation of fertility in Gorlin-Goltz syndrome seems problematic. We used a follow-up approach because our patient had a small tumor at the time of recurrence; however, subsequently, we observed an increasing trend in the tumor size.

There are case reports of ovarian fibrosarcoma developing in basal cell nevus 
syndrome [13]. In the present case, no malignant sign for ovarian tumor was revealed on the image. Thus, careful observation is required for follow-up of similar cases.

When we selected conservative observation, there was a risk of re-torsion and a chance that normal ovarian preservation by surgery could not be sufficiently performed after the tumor increased in size. Therefore, we presented tumorectomy for recurrent ovarian tumor as a choice to the patient. Because she had undergone right salpingo-oophorectomy and left ovarian tumorectomy previously, her AMH level was $1.53 \mathrm{ng} / \mathrm{mL}$. It was reported that the mean AMH level was $4.18 \mathrm{ng} / \mathrm{mL}$ in 1009 Chinese women aged 26 - 44 years [14]. Thus, our patient's AMH level was relatively low. Under this circumstance, when tumorectomy for recurrent ovarian tumor is implemented, premature ovarian failure onset may occur; thus, we decided to cryopreserve her oocytes before tumorectomy.

The AMH level and predicted response to ovulation induction in our patient were low. In a study on the ovulation induction method for poor responders, the difference in the number of MII oocytes retrieved was statistically significant between the short GnRH agonist protocol group and standard flexible GnRH antagonist protocol group and between the short $\mathrm{GnRH}$ agonist protocol group and clomiphene citrate plus gonadotropins in a flexible GnRH antagonist protocol group $(\mathrm{P}<0.015)$ [15]. Hence, we used the short GnRH protocol as the controlled ovarian stimulation protocol.

The survival rate of cryopreserved embryos after thawing in the ultra-rapid vitrification method is approximately 95\% [16] [17]. In contrast, the survival rate of cryopreserved oocytes after thawing is reported as $84.7 \%(2304 / 2721)$ [18]. There is a possibility that the survival rate after thawing may be slightly decreased in cryopreserved oocytes than in cryopreserved embryos. However, even a high survival rate after thawing can be expected in oocyte cryopreservation. In a randomized controlled trial comparing assisted reproductive technology, results of 4282 vitrified oocytes and 3524 fresh oocytes showed that ongoing pregnancy, embryo cleavage, and fertilization did not differ between the two [19].

In 2013, the American Society for Reproductive Medicine and the Society (ASRM) reported no increase in the incidence of chromosomal abnormalities and developmental disorders on examination of frozen egg-derived infants. Therefore, the ASRM issued a statement that oocyte vitrification and warming are no longer experimental procedures [20]. Moreover, the clinical pregnancy rate per thawed oocyte was reported to be from $4.5 \%$ to $12 \%$ [20]. On the basis of these data, about 10 eggs are thought necessary to obtain one child. With the limited time and cost and physical burden considered, it seemed very difficult to cryopreserve 10 eggs in the present case. A third egg collection could not be performed because the patient was unable to pay for the high cost of this treatment. In the prefecture of Japan where we live, oocyte cryopreservation for medical adaptation is not subject to subsidy from the administration, although 
government intervention is expected in the future.

Generally, ovum cryopreservation is performed before chemotherapy for patients with malignant disease, as a social adaptation, and in emergencies, such as when the husband's sperm cannot be obtained during egg collection. There are a few reports of oocyte cryopreservation before operative treatment of a benign ovarian tumor. In rare cases of Gorlin-Goltz syndrome wherein both ovarian tumors are detected at an early age and a low AMH level is observed, oocyte cryopreservation could be presented to the patient as an option of fertility preservation preoperatively. Although our patient preserved the possibility of pregnancy by cryopreserving her oocytes, conceiving a child naturally would have been most desirable at an early stage. In addition, as our patient has a risk of developing basal cell carcinoma, multiple treatments by experts from other fields are being continued.

\section{Acknowledgements}

We thank Ai Igarashi and Yukihiro Jinbo for performing the oocyte cryopreservation in the laboratory. We would also like to thank Editage (http://www.editage.com) for English language editing. The authors received no financial support for this study.

\section{Disclosures}

\section{Conflict of Interest}

Makoto Chihara, Atsushi Fujimoto, Kanna Ogi, Tatsuya Ishiguro, Kunihiko Yoshida, Masayuki Sekine, Koich Takakuwa, Hiroki Fujikawa, Yutaka Shimomura, and Takayuki Enomoto declare that they have no conflict of interest.

\section{Human Rights and Informed Consent}

All procedures followed were in accordance with the ethical standards of the responsible committee on human experimentation (institutional and national) and with the Helsinki Declaration of 1964 and its later amendments. Informed consent was obtained from the patient for inclusion in the study.

\section{Animal Studies}

This article does not contain any studies with animal subjects performed by the any of the authors.

\section{Approval by Ethics Committee}

Oocyte cryopreservation for this patient was approved by the Clinical Ethical Committee of Niigata University Medical and Dental Hospital.

\section{Clinical Trial Registry}

Not applicable. 


\section{Data Accessibility Statement}

All data related to this study are included in the manuscript.

\section{References}

[1] Gorlin, R.J. and Goltz, R.W. (1960) Multiple Nevoid Basal-Cell Epithelioma, Jaw Cysts and Bifid Rib. A Syndrome. The New England Journal of Medicine, 262, 908-912. https://doi.org/10.1056/NEJM196005052621803

[2] Manfredi, M., Vescovi, P., Bonanini, M. and Porter, S. (2004) Nevoid Basal Cell Carcinoma Syndrome: A Review of the Literature. International Journal of Oral \& Maxillofacial Surgery, 33, 117-124. https://doi.org/10.1054/ijom.2003.0435

[3] Gorlin, R.J. (1987) Nevoid Basal-Cell Carcinoma Syndrome. Medicine (Baltimore), 66, 98-113. https://doi.org/10.1097/00005792-198703000-00002

[4] Bonifas, J.M., Bare, J.W., Kerschmann, R.L., Master, S.P. and Epstein Jr., E.H. (1994) Parental Origin of Chromosome 9q22.3-q31 Lost in Basal Cell Carcinomas from Basal Cell Nevus Syndrome Patients. Human Molecular Genetics, 3, 447-448. https://doi.org/10.1093/hmg/3.3.447

[5] Hahn, H., Wicking, C., Zaphiropoulous, P.G., Gailani, M.R., Shanley, S., Chidambaram, A., et al. (1996) Mutations of the Human Homolog of Drosophila Patched in the Nevoid Basal Cell Carcinoma Syndrome. Cell, 85, 841-851. https://doi.org/10.1016/S0092-8674(00)81268-4

[6] Fujii, K., Kohno, Y., Sugita, K., Nakamura, M., Moroi, Y., Urabe, K., et al. (2003) Mutations in the Human Homologue of Drosophila Patched in Japanese Nevoid Basal Cell Carcinoma Syndrome Patients. Human Mutation, 21, 451-452. https://doi.org/10.1002/humu.9132

[7] Endo, M., Fujii, K., Sugita, K., Saito, K., Kohno, Y. and Miyashita, T. (2012) Nationwide Survey of Nevoid Basal Cell Carcinoma Syndrome in Japan Revealing the Low Frequency of Basal Cell Carcinoma. American Journal of Medical Genetics Part A, 158A, 351-357. https://doi.org/10.1002/ajmg.a.34421

[8] Fujii, K. and Miyashita, T. (2014) Gorlin Syndrome (Nevoid Basal Cell Carcinoma Syndrome): Update and Literature Review. Pediatrics International, 56, 667-674. https://doi.org/10.1111/ped.12461

[9] Kimonis, V.E., Goldstein, A.M., Pastakia, B., Yang, M.L., Kase, R., DiGiovanna, J.J., et al. (1997) Clinical Manifestations in 105 Persons with Nevoid Basal Cell Carcinoma Syndrome. American Journal of Medical Genetics, 69, 299-308. https://doi.org/10.1002/(SICI)1096-8628(19970331)69:3<299::AID-AJMG16>3.0.C $\underline{\mathrm{O} ; 2-\mathrm{M}}$

[10] Seracchioli, R., Bagnoli, A., Colombo, F.M., Missiroli, S. and Venturoli, S. (2001) Conservative Treatment of Recurrent Ovarian Fibromas in a Young Patient Affected by Gorlin Syndrome. Human Reproduction, 16, 1261-1263. https://doi.org/10.1093/humrep/16.6.1261

[11] Pirschner, F., Bastos, P.M., Contarato, G.L., Bimbato, A.C. and Filho, A.C. (2012) Gorlin Syndrome and Bilateral Ovarian Fibroma. International Journal of Surgery Case Reports, 3, 477-480. https://doi.org/10.1016/j.ijscr.2012.05.015

[12] Kurman, R.J. (1997) Blaustein's Pathology of the Female Genital Tract. 4th Edition, Springer, New York.

[13] Kraemer, B.B., Silva, E.G. and Sneige, N. (1984) Fibrosarcoma of the Ovary. A New Component in the Nevoid Basal-Cell Carcinoma Syndrome. The American Journal of Surgical Pathology, 8, 231-236. 
https://doi.org/10.1097/00000478-198403000-00010

[14] Loy, S.L., Cheung, Y.B., Fortier, M.V., Ong, C.L., Tan, H.H., Nadarajah, S., et al. (2017) Age-Related Nomograms for Antral Follicle Count and Anti-Mullerian Hormone for Subfertile Chinese Women in Singapore. PLoS One, 12, e0189830. https://doi.org/10.1371/journal.pone.0189830

[15] Schimberni, M., Ciardo, F., Schimberni, M., Giallonardo, A., De Pratti, V. and Sbracia, M. (2016) Short Gonadotropin-Releasing Hormone Agonist versus Flexible Antagonist versus Clomiphene Citrate Regimens in Poor Responders Undergoing In Vitro Fertilization: A Randomized Controlled Trial. European Review for Medical and Pharmacological Sciences, 20, 4354-4361.

[16] Mukaida, T. and Oka, C. (2012) Vitrification of Oocytes, Embryos and Blastocysts. Best Practice \& Research: Clinical Obstetrics \& Gynaecology, 26, 789-803.

https://doi.org/10.1016/j.bpobgyn.2012.07.001

[17] Rama Raju, G.A., Haranath, G.B., Krishna, K.M., Prakash, G.J. and Madan, K. (2005) Vitrification of Human 8-Cell Embryos, a Modified Protocol for Better Pregnancy Rates. Reproductive BioMedicine Online, 11, 434-437.

https://doi.org/10.1016/S1472-6483(10)61135-2

[18] Rienzi, L., Cobo, A., Paffoni, A., Scarduelli, C., Capalbo, A., Vajta, G., et al. (2012) Consistent and Predictable Delivery Rates after Oocyte Vitrification: An Observational Longitudinal Cohort Multicentric Study. Human Reproduction, 27, 1606-1612. https://doi.org/10.1093/humrep/des088

[19] Cobo, A. and Diaz, C. (2011) Clinical Application of Oocyte Vitrification: A Systematic Review and Meta-Analysis of Randomized Controlled Trials. Fertility and Sterility, 96, 277-285. https://doi.org/10.1016/j.fertnstert.2011.06.030

[20] Practice Committees of American Society for Reproductive Medicine, Society for Assisted Reproductive Technology (2013) Mature Oocyte Cryopreservation: A Guideline. Fertility and Sterility, 99, 37-43.

https://doi.org/10.1016/j.fertnstert.2012.09.028 